\title{
THE SELFADJOINT OPERATORS OF A VON NEUMANN ALGEBRA FORM A CONDITIONALLY COMPLETE LATTICE
}

\author{
MILTON PHILIP OLSON
}

\begin{abstract}
The bounded resolutions of the identity in a von Neumann algebra can be ordered by $\left\{E_{S}(u)\right\} \preceq\left\{E_{T}(u)\right\}$ if $E_{S}(u)$ $\geqq E_{T}(u), u \in R$. The selfadjoint operators in the algebra are partially ordered by this relation and are shown to form a conditionally complete lattice. The lattice operations are (essentially) defined by $E_{V S_{\alpha}}(u)=\wedge E_{S_{\alpha}}(u)$ for all $u$ contained in $R$. This order is called spectral order and agrees with the usual order on commutative subalgebras. For positive operators, $S$ is greater than or equal to $T$ in spectral order if and only if $S^{n}$ is greater than or equal to $T^{n}$ in the usual order for all $n \geqq 1$. Kadison's well-known counterexample is shown to fail. The operator lattice defined by spectral order differs from a vector lattice in the fact that $S \succeq T$ does not imply that $S+C \succeq T+C$.
\end{abstract}

1. Introduction. Let $a$ be a von Neumann algebra of operators on a Hilbert space $H$, and let $\delta$ be the real vector space of selfadjoint elements of $a . S \leqq T$ means

$$
(S x, x) \leqq(T x, x)
$$

for all $x \in H$. With this partial order, $S$ is a partially ordered vector space. Kadison [1] showed, long ago, that even when $a$ is the set of all bounded operators on $H, \mathcal{S}$ is not a complete vector lattice. In fact, Sherman [3] showed that if the selfadjoint operators in a $C^{*}$ algebra are lattice-ordered by the nonnegative elements, then the algebra is commutative.

The purpose of this paper is to report on an investigation of another order relation on $S$ with respect to which $S$ is conditionally complete. This order, called spectral order and denoted by " $\succeq$ ", is not a vector order on $S$. The missing property is that

$$
A-B \succeq 0
$$

Presented to the Society, January 24, 1969; received by the editors October 10, 1968 and, in revised form, November 10, 1969.

AMS 1970 subject classifications. Primary 46L10, 06A23; Secondary 46A40, 06A25.

Key words and phrases. von Neumann algebra, resolution of the identity, projection lattice, conditionally complete lattice, spectral integral.

Copyright (C) 1971, American Mathematical Society 
does not imply

$$
A \succeq B
$$

unless $A$ and $B$ commute. In that case, $A \geqq B$ is equivalent to $A \succeq B$. For positive operators, $A \succeq B$ if and only if $A^{p} \geqq B^{p}$ for all integers $p \geqq 1$. This paper is a revision and extension of another paper having the same title which was circulated privately.

2. Resolutions of the identity. Let $\odot$ be the selfadjoint projections in $a$. It is known that each element of $\mathcal{S}$ finds its spectral resolution in $\beta$ and that $\beta$ is a complete sublattice of the complete lattice of all projections in $H$. The latter statement means that the supremum of a family in $\rho$ in the lattice of all projections is an element of $P$. A resolution of the identity in $Q$ is a right-continuous isotone map $T$ of the line $R$ into $P$ such that there exists $m>0$ such that

(2.1) $T(u)=0, u<-m$ and

(2.2) $T(u)=I, u>m$.

By the uniqueness assertion of the spectral theorem, it is known that $\mathcal{e}$, the set of resolutions of the identity in $a$, is in one-to-one correspondence with $S$.

Definition 1. Let $S_{1}$ and $S_{2}$ be selfadjoint elements of the von Neumann algebra $a$. Let $T_{1}$ and $T_{2}$ be the resolutions of the identity of $S_{1}$ and $S_{2}$, respectively. $S_{1} \preceq S_{2}$ if

$$
T_{1}(u) \geqq T_{2}(u), \quad u \in R .
$$

The order " $\preceq$ " will be called spectral order. (It is clear that spectral order is partial order in $S$.)

Lemma 1. Let $\left\{T_{\alpha}\right\}_{\alpha \in A} \subset \mathcal{C}$ and suppose there exists $T \in \mathcal{C}$ such that

$$
T(u) \leqq T_{\alpha}(u), \quad u \in R, \alpha \in A .
$$

Define

$$
W(u)=\bigwedge_{\alpha \in A} T_{\alpha}(u), \quad u \in R .
$$

Then $W \in \mathfrak{e}$. If $Y \in \mathfrak{e}$ is such that

$$
W(u) \leqq Y(u) \leqq T_{\alpha}(u), \quad u \in R, \alpha \in A,
$$

then

$$
W=Y \text {. }
$$

Proof. Let $m$ be the bound on $T$ required in (2.1) and (2.2). Let $m_{\alpha}$ be the bound for $T_{\alpha}$ for some $\alpha \in A$. Then, $W(u)=0, u<-m_{\alpha}$, and 
$W(u)=I$ for $u>m$. Thus, $W$ has a bound $\max \left\{m, m_{\alpha}\right\} . W$ is clearly an isotone map of $R$ into $P$.

$W$ is right-continuous. For,

$$
\begin{aligned}
\bigwedge_{v>u} W(v) & =\bigwedge_{v>u}\left(\bigwedge_{\alpha \in A} T_{\alpha}(v)\right)=\bigwedge_{\alpha \in A}\left(\bigwedge_{v>u} T_{\alpha}(v)\right) \\
& =\bigwedge_{\alpha \in A} T_{\alpha}(u)=W(u) .
\end{aligned}
$$

Thus, $W \in \mathfrak{C}$. The last assertion is clear from the definition of $W$. Q.E.D.

If $T$ is any isotone map of $R$ into $\mathcal{P}$, then $T$ is called bounded if it satisfies (2.1) and (2.2) for some finite number $m>0$.

LEMMA 2. If $T$ is a bounded isotone map of $R$ into $P$, then $T$ determines a unique element $T_{r} \in \mathcal{C}$ by defining

$$
T_{r}(u)=\bigwedge_{v>u} T(v)
$$

$T$, is called the right-regularization of $T$.

Proof. Let $\epsilon>0$ be any positive number. If $m$ is a bound for $T$, then $m+\epsilon$ is a bound for $T_{r} . T_{r}$ is an isotone map of $R$ into $P$. It remains to show that $T_{r}$ is right-continuous.

$$
\bigwedge_{v>u} T_{r}(v)=\bigwedge_{v>u} \bigwedge_{w>v} T(w)=\bigwedge_{w>u} T(w)=T_{r}(u)
$$

$T_{r} \in$ e. Q.E.D.

LemmA 3. Let $\left\{T_{\alpha}\right\}_{\alpha \in A} \subset \mathcal{C}$ and suppose there exists $T \in \mathcal{C}$ such that

$$
T(u) \geqq T_{\alpha}(u), \quad u \in R, \alpha \in A .
$$

Define

$$
V(u)=\bigvee_{\alpha \in A} T_{\alpha}(u), \quad u \in R .
$$

Then $V$ is a bounded isotone map of $R$ into $\odot$. $V_{r}$, the right-regularization of $V$, is contained in $\mathfrak{C}$ and has the property that if $Y \in \mathcal{C}$ and

$$
V_{r}(u) \geqq Y(u) \geqq T_{\alpha}(u), \quad u \in R, \alpha \in A,
$$

then

$$
V_{r}=Y
$$

Proof. $V$ is a bounded isotone map by arguments analogous to those used in the proof of Lemma 1. $V_{r} \in \mathcal{C}$ by Lemma $2 . V_{r}(u) \geqq V(u)$ 
by the definition of the right-regularization given in equation (2.9). It follows that

$$
V_{r}(u) \geqq Y(u) \geqq V(u), \quad u \in R .
$$

But then, for all $u \in R$,

$$
Y(u) \leqq V_{r}(u)=\bigwedge_{v>u} V(v) \leqq \bigwedge_{v>u} Y(v)=Y(u) .
$$

So $Y=V_{r}$. Q.E.D.

THEOREM $1 . S$ is a conditionally complete lattice under the partial order " $\succeq$ ".

Proof. In Lemmas 1, 2, and 3, it has been shown that $\mathcal{C}$ is a conditionally complete lattice under the reverse of the product order determined by the order structure in $\mathcal{P}$. $\mathcal{C}$ is in one-to-one correspondence with $\delta$; and the order " $\succeq$ " in $\delta$ is by definition the aforementioned order in C. Q.E.D.

3. Properties of spectral order. The purpose of this section is to compare and contrast spectral order with the usual order on selfadjoint operators. An element $T \in \mathcal{S}$ is nonnegative in spectral order if $T \succeq 0$. By definition, the spectrum of $T$ is nonnegative; and, hence, $T \geqq 0$ in the usual order. Conversely, $T \geqq 0$ implies $T$ has nonnegative spectrum and so $T \succeq 0$. The two orders, therefore, have the same positive cones.

It is known that $(\delta, \geqq)$ is an ordered vector space, and that the order is completely determined by the positive cone. If $(s, \succeq)$ were an ordered vector space, then, since it shares the same positive cone with $(s, \geqq)$, it would be the same order. We shall show below that $(s, \succeq)$ is not an ordered vector space and that " $\succeq$ " is distinct from " $\geqq$ ".

Lemma 4. Let $S, T \in S$ and $S \succeq T$. Then $S \geqq T$.

Proof. Let

$$
f_{n}=\sum_{i=1}^{p_{n}} \alpha_{i n} \chi_{E_{i n}}
$$

be a sequence of nonnegative nondecreasing simple functions converging monotonely upward to the function $f(u)=u$. We may assume that these simple functions are constructed on a partition of $[-m, m]$ into subintervals of length $2 m / n$, where $m$ is some number greater 
than both $\|S\|$ and $\|T\|$. Then, clearly,

$$
f_{n}(S) \uparrow S
$$

and

$$
f_{n}(T) \uparrow T
$$

But

$$
\left(f_{n}(T) x, x\right) \leqq\left(f_{n}(S) x, x\right), \quad x \in H,
$$

as direct calculation easily shows. Hence,

$$
(S x, x) \geqq\left(f_{n}(S) x, x\right) \geqq\left(f_{n}(T) x, x\right), \quad n=1,2, \cdots .
$$

Taking the limit on the right-hand side, we have

$$
(S x, x) \geqq(T x, x), \quad x \in H . \quad \text { Q.E.D. }
$$

TheOREM 2. Let $S, T \in S$. If $S$ and $T$ commute, $S \succeq T$ is equivalent to $S \geqq T$.

Proof. By Lemma 4, $S \succeq T$ implies $S \geqq T$. Conversely, suppose $S \geqq T$. Since $S$ and $T$ commute by hypothesis, the families $\left\{E_{S}(u)\right\}_{u \in R}$ and $\left\{E_{T}(u)\right\}_{u \in R}$ commute. If $S \succeq T$ does not hold, there exists $u \in R$ such that

$$
E_{S}(u) E_{T}(u)=E_{T}(u) E_{S}(u)<E_{S}(u) .
$$

This means that there exists an $x \neq 0$ such that

$$
x \in E_{S}(u)\left(I-E_{T}(u)\right) H \text {. }
$$

According to Theorem 5 in Lengyel and Stone's paper [2],

$$
(S x, x) \leqq u(x, x)
$$

since $u$ is the least upper bound of the form $(S x, x)$ on $E_{S}(u) H$. On the other hand,

$$
(T x, x)>u(x, x)
$$

because $x \in\left(I-E_{T}(u)\right) H$ and $u$ is the greatest lower bound of the form $(T x, x)$. Q.E.D.

Finally, spectral order can be characterized in terms of the usual order.

THEOREM 3. Let $A$ and $B$ be bounded positive selfadjoint operators on $H$. Then $A \succeq B$ if and only if $A^{p} \geqq B^{p}$ for $p=1,2, \cdots$.

Proof. According to Lengyel and Stone [2], if $A$ is a bounded 
positive selfadjoint operator, then for each $u \in R$ the spectral projection $E_{A}(u)$ is the projection on the closed linear manifold

$$
\mathfrak{M}_{A}(u)=\left\{x \mid\left\|A^{p} x\right\| \leqq u^{p}\|x\|, p \geqq 1\right\} .
$$

But,

$$
\left\|A^{p} x\right\|^{2}=\left(A^{2 p} x, x\right) \geqq\left(B^{2 p} x, x\right)=\left\|B^{p} x\right\|^{2} .
$$

Hence,

$$
\mathfrak{M}_{B}(u) \supset \mathfrak{T}_{A}(u)
$$

and

$$
E_{B}(u) \geqq E_{A}(u), \quad u \in R .
$$

This implies, by definition,

$$
A \succeq B .
$$

Conversely, suppose $A \succeq B$. We wish to show that

$$
\int u^{p}\left(E_{A}(d u) x, x\right) \geqq \int u^{p}\left(E_{B}(d u) x, x\right)
$$

for all $x \in H$ and $p=1,2, \cdots$. Exactly the same sequence of simple functions mentioned in the proof of Lemma 4 can be employed to show that

(3.18) $\left(f_{n}^{p}(A) x, x\right) \uparrow\left(A^{p} x, x\right)$ and

(3.19) $\left(f_{n}^{p}(B) x, x\right) \uparrow\left(B^{p} x, x\right)$

for all $x \in H$ and $p=1,2, \cdots$. Because of the nondecreasing character of $f_{n}^{p}$ as a function of $u \in R$, it is easy to show by direct calculation that

$$
\left(f_{n}^{p}(A) x, x\right) \geqq\left(f_{n}^{p}(B) x, x\right)
$$

for $x \in H$ and $p=1,2, \cdots$ (3.17) follows by taking limits on $n$. Q.E.D.

CoRollary 1. Let $A$ and $B$ be bounded positive selfadjoint operators on $H$. Let $D$ be a bounded closed interval which contains the spectra of both $A$ and $B$. Then $A \succ B$ if and only if for all $f$ continuous and nondecreasing on $D, f(A) \geqq f(B)$.

Proof. $u^{p}$ is continuous and nondecreasing on $D$ for $p=1,2, \cdots$. This implies the sufficiency of the condition $f(A) \geqq f(B)$. The proof of necessity follows by exactly the same approximation argument used in the proof of the theorem. Q.E.D.

Finally, let us show that spectral order and the usual order are 
distinct. The following example is essentially due to Kadison [1]. Let $H$ be a three-dimensional complex Hilbert space with or thogonal basis $\left\{e_{1}, e_{2}, e_{3}\right\}$. Let $M$ be the closed subspace spanned by $e_{1}$ and $e_{2} . P_{i}$ is projection on $e_{i}$ for $i=1,2,3$. $Q$ is projection on $M$. Let $A$ be the operator on $M$ whose matrix is given by

$$
A=\left(\begin{array}{cc}
-1 & 2^{1 / 2} \\
2^{1 / 2} & -1
\end{array}\right)
$$

The selfadjoint operator $A Q$ is neither positive nor negative in the usual order of selfadjoint operators. Thus, it is neither bounded above nor below by the zero operator. $P_{1}$ and $P_{2}$ are commuting orthogonal projections. By the remark above, they have infimum 0 in spectral order. Suppose that 0 was the infimum in the usual order. A contradiction is reached if it can be shown that $A Q$ is less than $P_{1}$ and $P_{2}$ in the usual order; for, in that case, 0 is not the greatest of the lower bounds since $A Q$ and 0 are not even comparable.

In matrix form on $H$,

$$
P_{1}-A Q=\left(\begin{array}{lll}
2 & 2^{1 / 2} & 0 \\
2^{1 / 2} & 1 & 0 \\
0 & 0 & 0
\end{array}\right) .
$$

By the usual test, this is a positive matrix and so defines a positive operator. In fact, the eigenvalues are $\lambda=0$ and $\lambda=3$. Because the usual order is a vector order $P_{1}-A Q \geqq 0$ if and only if $P_{1} \geqq A Q$. Similar calculations show that $P_{2} \geqq A Q$. Therefore, 0 is not the infimum in the usual order and so the two orders are distinct.

By remarks above, it is certainly true that $P_{1}-A Q \succ 0$. Let us show that $P_{1} \succeq A Q$ does not hold. The spectral resolution of $P_{1}$ is given by

(3.23) $E(u)=0,-\infty<u<0$;

(3.24) $E(u)=P_{2}+P_{3}, 0 \leqq u<1$;

(3.25) $E(u)=I, 1 \leqq u<\infty$.

The eigenvalues of $A Q$ are $\lambda=-1-2^{1 / 2}, 0$, and $-1+2^{1 / 2}$. To the eigenvalue $\lambda=-1-2^{1 / 2}$ corresponds the projection on the subspace of vectors of the form

$$
x=\left(\begin{array}{c}
a \\
-a \\
0
\end{array}\right),
$$

where $a$ is any complex number. Call this projection $Q_{1} . P_{3}$ is clearly projection on the subspace corresponding to the eigenvalue $\lambda=0 . Q_{3}$, 
projection on the subspace of vectors of the form

$$
x=\left(\begin{array}{l}
a \\
a \\
0
\end{array}\right),
$$

corresponds to the eigenvalue $\lambda=-1+2^{1 / 2}$. Therefore, the resolution of the identity for $A Q$ is given by

(3.28) $F(u)=0,-\infty<u<-1-2^{1 / 2}$;

(3.29) $F(u)=Q_{1},-1-2^{1 / 2} \leqq u<0$;

(3.30) $F(u)=Q_{1}+P_{3}, 0 \leqq u<-1+2^{1 / 2}$;

(3.31) $\quad F(u)=I,-1+2^{1 / 2} \leqq u<\infty$.

It is easy to see that $F(0) H$ neither contains nor is contained in $E(0) H$. Hence, $P_{1} \succeq A Q$ does not hold.

\section{BiBLIOGRAPHY}

1. R. Kadison, Order properties of bounded self-adjoint operators, Proc. Amer. Math. Soc. 2 (1951), 505-510. MR 13, 47.

2. B. Lengyel and M. Stone, Elementary proof of the spectral theorem, Ann. of Math. (2) 37 (1936), 853-864.

3. S. Sherman, Order in operator algebras, Amer. J. Math. 73 (1951), 227-232 MR 13, 47.

University of California, Davis, California 95616 